\title{
Separable Hilbert space for loop quantization
}

\author{
J. Fernando Barbero G., ${ }^{1,2, *}$ Tomasz Pawłowski, ${ }^{3,4, \dagger}$ and Eduardo J. S. Villaseñor ${ }^{5,2, \$}$ \\ ${ }^{1}$ Instituto de Estructura de la Materia, CSIC, Serrano 123, 28006 Madrid, Spain \\ ${ }^{2}$ Grupo de Teorías de Campos y Física Estadística, Instituto Universitario Gregorio Millán Barbany, \\ Universidad Carlos III de Madrid, Unidad Asociada al IEM-CSIC \\ ${ }^{3}$ Departamento de Ciencias Físicas, Facultad de Ciencias Exactas, Universidad Andres Bello, \\ República 220, Santiago 8370134, Chile \\ ${ }^{4}$ Wydziat Fizyki, Uniwersytet Warszawski, Hoża 69, 00-681 Warszawa, Poland \\ ${ }^{5}$ Instituto Gregorio Millán, Grupo de Modelización y Simulación Numérica, \\ Universidad Carlos III de Madrid, Avda. de la Universidad 30, 28911 Leganés, Spain
}

(Received 25 March 2014; published 24 September 2014)

\begin{abstract}
We discuss, within the simplified context provided by the polymeric harmonic oscillator, a construction leading to a separable Hilbert space that preserves some of the most important features of the spectrum of the Hamiltonian operator. This construction may be applied to other polymer quantum mechanical systems, including those of loop quantum cosmology, and is likely generalizable to certain formulations of full loop quantum gravity. It is helpful to sidestep some of the physically relevant issues that appear in that context, in particular those related to superselection and the definition of suitable ensembles for the statistical mechanics of these types of systems.
\end{abstract}

DOI: 10.1103/PhysRevD.90.067505

\section{INTRODUCTION AND PRELIMINARY REMARKS}

Canonical loop quantum gravity [1,2] (LQG) is at present one of the most advanced approaches to address the quantization of general relativity. Recent progress in this field, involving in particular the application of deparametrization techniques with respect to the matter frames [3-5], has brought LQG to a level which makes it possible to probe its dynamical predictions [5].

Despite this progress, some critical problems of the theory remain open. One of the principal issues is the construction of suitable Hilbert space(s), which is essential to extract dynamical predictions out of the models. The standard constructions lead to an orthonormal basis labeled by spin-networks-graphs embedded in three-dimensional differential manifold with colored edges and vertices [1]. Unfortunately, the noncountable number of spin networks renders the Hilbert space nonseparable. This feature creates some difficulties in the development of the formalism both to define unitary evolution and to build suitable statistical ensembles. This problem arises both at the level of the full theory and in its symmetry reduced quantum-mechanical versions [6], in particular the ones applied in loop quantum cosmology (LQC) [7].

This issue has been substantially addressed in the LQG literature over the years (see for example the review [8]). The attempts on solving it start with a proposal presented by Zapata in [9] where a method that avoids the nonseparability of the LQG Hilbert space by using piecewise

\footnotetext{
*fbarbero@iem.cfmac.csic.es

tomasz.pawlowski@unab.cl

¥ejsanche@math.uc3m.es
}

PACS numbers: 04.60.Pp, 04.60.Ds, 03.65.Ca, 03.65.Db

linear graphs in a piecewise linear manifold is proposed. Other ideas in the same direction appear in [10] where, as a cure to nonseparability, the authors extended the diffeomorphism group and, hence, enlarged the group of gauge transformations by allowing them to act as homeomorphisms at spin network nodes.

There is however no consensus about these proposals within the general community as (i) necessary modifications to the underlying classical framework deviate too far from general relativity [8] and (ii) their inclusion could deprive the theory of certain desirable properties. In particular it is not known how the proposal of [10] would mesh with the strong uniqueness theorems [11] about the representation of the kinematical algebra of basic observables in LQG or with the absence of a classical counterpart of the proposed enlarged symmetry group.

The purpose of this article is to present a construction of the physical Hilbert space used in loop quantization (in particular in LQC) which avoids the nonseparability issues while retaining the correct low energy (large scale) behavior of the resulting framework. The construction is exemplified in the particular case of the polymer quantum harmonic oscillator. This particular system is of critical relevance to inhomogeneous LQC frameworks as the harmonic oscillator is the main building block of the Fock spaces representing the inhomogeneity modes [12,13], thus its loop quantization is a necessary step to go beyond the hybrid quantization scheme $[12,14]$ (which is the core of almost all the present treatments of inhomogeneous scenarios in LQC $[13,14])$ and implementing the loop quantization to all degrees of freedom (see for example the construction in [15]).

This particular system (the polymer quantized harmonic oscillator) has been recently analyzed in [6]. The conclusion 
of that analysis is that the treatments presented up to date in the literature were insufficient, especially for the applications listed in the paragraph above. However, no suitable solution to the problem has been found there. In our present work we fill this gap, providing the precise construction of a suitable (separable) Hilbert space. An important consequence of the analysis presented in this article is the possibility of defining suitable statistical ensembles appropriate for the discussion of thermodynamical problems for these kinds of systems.

The construction that we provide here is based on the use of certain "foliations" of the original nonseparable Hilbert spaces by means of separable subspaces and a natural Lebesgue measure on it. This construction follows from observations of [16] and was successfully applied in [17] to the case of the polymer-quantized scalar field.

We will illustrate the procedure that we suggest in the case of the harmonic oscillator quantized via polymer techniques as specified in [6].

\section{POLYMERIC QUANTUM HARMONIC OSCILLATOR}

Let us start by briefly recalling the quantization procedure and the features of the polymer harmonic oscillator leading to the problems indicated in [6].

The main properties of the system are as follows:

(1) The Hilbert space is the (nonseparable) space of square integrable functions on the Bohr compactification of the real line $\mathcal{H}=L^{2}\left(\mathbb{R}_{\mathrm{Bohr}}, \mathrm{d} \mu\right)$.

(2) The spectrum of the Hamiltonian features a (continuous) band structure; however, it remains a pure point spectrum.

Classically, the time evolution of the harmonic oscillator is generated by the Hamiltonian

$$
\boldsymbol{H}(q, p)=\frac{\hbar^{2}}{2 m \ell^{2}} p^{2}+\frac{m \ell^{2} \omega^{2}}{2} q^{2},
$$

where the canonical variables $p, q$ are dimensionless, while $\ell$ and $\omega$ are the oscillator's characteristic length and frequency respectively.

In loop quantization it is impossible to promote $p$ and $q$ to operators simultaneously. Among the infinite number of nonequivalent representations of the Weyl algebra in nonseparable Hilbert spaces there are two natural nonequivalent choices in the context of quantum cosmology: the position representation where the operator $\hat{q}$ is well defined, and the momentum one, where $\hat{p}$ is well defined. In both representations the remaining variable has to be approximated ("regularized") in terms of other Weyl algebra elements and then promoted to be an operator.

To focus our attention we choose the position representation. A similar procedure works for the momentum one. In order to regularize the momentum we approximate it by using $V(q)=e^{-i p q}$. Thus the quantum Hamiltonian takes the form

$$
\hat{\boldsymbol{H}}=\frac{\hbar^{2}}{2 m\left(2 q_{0} \ell\right)^{2}}\left(2 \llbracket-\hat{V}\left(2 q_{0}\right)-\hat{V}\left(-2 q_{0}\right)\right)+\frac{m \ell^{2} \omega^{2}}{2} \hat{q}^{2},
$$

where $q_{0}$ is a regularization constant. The quantity $q_{0} \ell$ can be interpreted as a polymer scale.

The above Hamiltonian, when acting on the physical states represented respectively by the wave function $\tilde{\Psi} \in L^{2}\left(\mathbb{R}_{\mathrm{Bohr}}, \mathrm{d} \mu\right)$, or in terms of its Fourier-Bohr transform $\Psi \in \ell^{2}(\mathbb{R})$ [where $\ell^{2}(\mathbb{R})$ is the space of square summable functions on $\mathbb{R}$ ] can be written as a difference operator in $q$ and a differential one in $p$

$$
\begin{aligned}
{[\hat{\boldsymbol{H}} \Psi](q)=} & \frac{\hbar^{2}}{8 m q_{0}^{2}}\left(2 \Psi(q)-\Psi\left(q+2 q_{0}\right)-\Psi\left(q-2 q_{0}\right)\right) \\
& +\frac{m \ell^{2} \omega^{2}}{2} q^{2} \Psi(q), \\
{[\hat{\boldsymbol{H}} \tilde{\Psi}](p)=} & -\frac{m \ell^{2} \omega^{2}}{2} \tilde{\Psi}^{\prime \prime}(p)+\frac{\hbar^{2}}{2 m\left(q_{0} \ell\right)^{2}} \sin ^{2}\left(q_{0} p\right) \tilde{\Psi}(p) .
\end{aligned}
$$

The eigenvalue problem $\hat{\boldsymbol{H}} \tilde{\Psi}=E \tilde{\Psi}$ defined by (2.3b) takes the form of the Mathieu equation and the differential symbols appearing in the Hamiltonian have the same form of the ones describing a particle in periodic potential - a case well studied in the literature (see [18] for relevant mathematical details). We have to remember, however, that here the Hilbert space is different (in particular nonseparable).

If we consider the form of the Hamiltonian specified via (2.3a) it is a difference operator coupling the points separated by $2 q_{0}$. One can thus divide the domain of $\Psi(q)$ onto the set of uniform lattices - sets preserved by the action of $\hat{\boldsymbol{H}}$

$$
\mathbb{R}=\bigcup_{\epsilon \in[0,1)} \mathcal{L}_{\epsilon}, \quad \mathcal{L}_{\epsilon}:=2 q_{0}(\epsilon+\mathbb{Z}) .
$$

This observation has led to the solution presented in [19]. Since the lattices are preserved by the time evolution we can treat the subspaces $\mathcal{H}_{\epsilon}$ spanned by the cutoff of the wave function support to a single $\mathcal{L}_{\epsilon}$ as "superselection" sectors. The customary way to proceed in such case is to select the single sector (represented by a single value of $\epsilon$ ) and work just with it. This approach has been applied, for example, in LQC [20,21].

Under this choice, the Hilbert space $\mathcal{H}$ gets restricted to a subspace $\mathcal{H}_{\epsilon}$ defined by the projection $\mathcal{H} \ni \tilde{\Psi} \mapsto \tilde{\Psi}_{\epsilon}=$ $\left.\tilde{\Psi}\right|_{\mathcal{L}_{\epsilon}} \in \mathcal{H}_{\epsilon}$. The subspace $\mathcal{H}_{\epsilon}$ is then a space of quasiperiodic functions of $p$ satisfying

$$
\tilde{\Psi}_{\epsilon}\left(p+\pi / q_{o}\right)=e^{-2 \pi i \epsilon} \tilde{\Psi}_{\epsilon}(p) .
$$

Such subspace is homeomorphic to a space of square integrable functions (in momentum representation) on a 
unit circle $L^{2}\left(S^{1}, \mathrm{~d} p\right)$ with the gluing (boundary) conditions depending on $\epsilon$. In particular, the case $\epsilon=0$ corresponds to periodic conditions, whereas $\epsilon=1 / 2$ corresponds to the antiperiodic ones. The spectrum $S$ of the Hamiltonian $\hat{\boldsymbol{H}}$ is a point spectrum and can be written as the union $S=\cup_{\epsilon} S_{\epsilon}$.

On the other hand, the reasoning presented in Sec. 4 of [17] and references therein shows that - in the context of LQC - a similar approach based on working within the subspaces $S_{\epsilon}$ may be problematic because the dynamics may connect different sectors. To avoid this kind of problem one should take into account all the sectors. In the case of the polymer harmonic oscillator this means that all the points of the bands describing the spectrum must be considered as for the particle in periodic potential in standard (Schrödinger) quantization. Notice, however, that the spectrum remains a pure point one despite having an uncountable number of elements. This immediately implies the nonseparability of the physical Hilbert space (constructed through the spectral decomposition of $\hat{\boldsymbol{H}}$ ) which is not a surprise as it should be equivalent to a nonseparable $L^{2}\left(\mathbb{R}_{\mathrm{Bohr}}, \mathrm{d} \mu\right)$. This severely hinders the application of this construction to analyze the physical properties of the loop quantum harmonic oscillator, in particular the statistical mechanics of the system as explained in detail in [6].

\section{INTEGRAL HILBERT STRUCTURE}

Nonseparability is a source of problems for some systems of physical interest related to LQC, in particular in the polymer quantization of the scalar field as discussed in [17]. There, the time dependence of the "lattice gap" causes a mixing of the putative "superselection" sectors during the time evolution, thus preventing one from working with just one superselected subspace. This feature seems to be a generic one in LQC models beyond the isotropic ones. On the other hand, in the case of the flat anisotropic Bianchi I universe with massless scalar field one can show, using the spectral properties of the evolution operator for the model, that the single sector Hilbert spaces do not admit a semiclassical sector [22].

In the context of LQC a possible solution to the problem has been presented in Appendix $\mathrm{C}$ of [23]. There the action of the evolution operator (playing the role of a Hamiltonian) is introduced via an action of its adjoint on a (bigger) dual to the original Hilbert space. Next one projects onto a single superselection space (this is known as the shadow states technique [24]). Finally the dual space is equipped with a postulated inner product defined by a Schrödinger quantization.

\section{A. The construction}

Here we present a systematic construction of a separable Hilbert space for LQC in terms of an integral of superselection sector Hilbert spaces: " $\overline{\mathcal{H}}=\int_{[0,1)} \mathcal{H}_{\epsilon} \mathrm{d} \epsilon$ " with the induced scalar product making it separable. The specific construction is inspired by the Hilbert space structures observed in LQC in the presence of a positive cosmological constant: more precisely the dependence of these structures on the lapse function [16]. The goal of that work was to construct the physical Hilbert space generated by the Hamiltonian constraint (isotropic and flat FriedmannLemaître-Robertson-Walker background with a scalar field source) through group averaging for various choices of the lapse $N$. Two examples, leading to distinct results, were considered: (i) $N=a^{3}$ (where $a$ is a scale factor) and (ii) $N=1$. In (i) the Hamiltonian constraint admits a 1-parameter family of self-adjoint extensions. Each extension has a discrete spectrum consisting of isolated points. In (ii) the Hamiltonian constraint admits a unique extensionits spectrum is purely continuous (well-defined Lebesgue measure). As a set, the spectrum is the union of the spectra of all the extensions found in the case (i).

By comparing the inner product structures in the Hilbert space $\overline{\mathcal{H}}$ constructed in the case $N=1$ with the ones that appear in each extension $\mathcal{H}_{\beta}$ of the case $N=a^{3}$ we notice that

$$
\overline{\mathcal{H}}=\int \mathcal{H}_{\beta} \mathrm{d} \sigma(\beta), \quad\langle\Psi \mid \chi\rangle_{\overline{\mathcal{H}}}=\int\left\langle\Psi_{\beta} \mid \chi_{\beta}\right\rangle_{\mathcal{H}_{\beta}} \mathrm{d} \sigma(\beta),
$$

where $\Psi_{\beta}(\omega):=\left.\Psi(\omega)\right|_{\omega^{2} \in \operatorname{Sp}(\boldsymbol{H})}$ and the measure $\mathrm{d} \sigma$ is induced by the Lebesgue measure on the spectrum of the constraint for $N=1$.

Following the previous observation, and noticing that the set of the $\epsilon$-lattice labels is Lebesgue measurable, we suggest one to introduce in LQC an analogous structure in terms of the superselection sectors $S_{\epsilon}$, by defining

$$
\overline{\mathcal{H}}:=\int_{[0,1)} \mathcal{H}_{\epsilon} \mathrm{d} \epsilon, \quad\langle\Psi \mid \chi\rangle_{\overline{\mathcal{H}}}=\int_{[0,1)}\left\langle\Psi_{\epsilon} \mid \chi_{\epsilon}\right\rangle_{\mathcal{H}_{\epsilon}} \mathrm{d} \epsilon,
$$

where $\Psi_{\epsilon}=\left.\Psi\right|_{\mathcal{L}_{\epsilon}}$. Notice that the measure $\mathrm{d} \epsilon$ can be replaced by $f(\epsilon) \mathrm{d} \epsilon$ (that takes into account the density of states in a suitable way) giving a unitarily equivalent Hilbert space structure.

In the particular case of the polymeric harmonic oscillator the resulting Hilbert space $\overline{\mathcal{H}}$ is mathematically equivalent to the one appearing in the Schrödinger quantization of a particle in a periodic potential. The main difference is that we have the standard band structure and the spectrum of $\boldsymbol{H}$ is purely continuous. As a consequence all the standard quantum mechanical tools can be used and, in particular, the quantum statistical mechanics of the system can be studied by following the usual approach.

It is worth noting that in the Schrödinger quantization of the particle in a periodic potential present in (2.3b) the Hilbert space admits a natural fiber bundle decomposition [18], the fibers of which are exactly the spaces $\mathcal{H}_{\epsilon}$ specified earlier. In the polymer quantization considered here the fiber 
structure is not present. Our construction can be seen as a recomposition of the (original) Hilbert space so that $\mathcal{H}_{\epsilon}$ are its fibers, using the natural Lebesgue measure on the space of superselection sector labels. The construction can be applied directly to existing models of isotropic (FRW) universe in LQC, as the structure of superselection sectors (in particular the topology of the space of sectors) is the same as in presented example. For example when applied to the flat FRW universe with massless scalar field it gives a result equivalent to construction specified in Appendix C of [23].

\section{B. Comparison with previous constructions}

A word of caution is necessary here. While in order to deal with the mixing of lattices by time evolution described in [17] it was convenient to introduce a separable Hilbert space as above, it is not clear why we cannot just consider a single superselection sector in the context of the polymer harmonic oscillator. This question is of particular relevance for LQC as the latter approach is, precisely, the one that has been followed there. Technically, as long as the "polymerization scale" $q_{0}$ is constant in time, restricting the quantum dynamics to a single superselection sector is both correct and consistent. The situation changes if we allow $q_{0}$ to be time dependent. In such a case, similarly to what happens in [17], one expects to have the phenomenon of "sector mixing" and then something should be done in order to avoid the problems associated with nonseparability, for instance, using the construction described before. This observation may be relevant for the loop quantization of the inhomogeneity modes in LQC as an explicit time dependence naturally arises there [14]. If one wants to have a uniform treatment for all the relevant cases, one should also follow the same approach when the polymerization scale is a constant. One has to understand, however, how the different choices can affect the physical results. In the studies of isotropic universes in LQC the dependence on the choice of the superselection sector has been systematically analyzed (see for example [21,25]). The differences in exact physical predictions appeared to be minor (confined to dispersion differences in the scattering picture [26] and the fine details of the near-bounce dynamics [27]), especially when appropriate quantization prescriptions were chosen [27]. Furthermore, for models with noncompact spatial slices the discrepancies vanished in the infrared regulator removal limit (see the discussion in [28]), whereas for the compact ones the differences became relevant only for "very quantum," physically uninteresting universes [29]. Since the sectors (the "fibers" in our approach) are orthogonal to each other, these features transfer directly to the theory arising from the construction proposed here. Thus, at least in the case of models studied so far, one can safely work with just one superselection sector without introducing significant errors in physical predictions as long as that choice does not violate the consistency of the model.

\section{GENERALIZATIONS AND OUTLOOK}

The construction presented here can be applied in a straightforward way to more general models within LQC featuring quasiglobal degrees of freedom. This is so because in many such models (see for example [30]) there is a natural division into a family of separable superselection sectors $\mathcal{H}_{\lambda}$ with $\lambda$ belonging to a set that can be equipped with the Lebesgue measure. However, the present construction may be relevant not only for simplified cosmological models but also for full LQG. Finding a suitable separable space is still an open problem. Our construction appears to be applicable at least in some of the approaches to the theory featuring spin network graphs of fixed topology, a feature present for example in algebraic quantum gravity [31].

In the standard formulation of LQG the Hilbert spaces are spanned by states supported on (piecewise analytic) graphs embedded in a differential manifold. The disjoint graphs are orthogonal, which together with (at least) continuum number of the graphs leads directly to the conclusion, that any such Hilbert space is nonseparable. If the action of the Hamiltonian constraint (or suitable deparametrized Hamiltonian) is graph preserving the functions supported on the particular single graph can be treated as a superselection sector (provided of course that all the observables used in the description are also graph preserving operators). Given that, one can construct the integral Hilbert space applying directly the technique introduced in this article, that is build the separable Hilbert space of which the distinguished superselection sectors are single fibers. However for this step it is essential to equip the family of the superselection sectors with a Lebesgue measure. While at present there is no indication of any significant problem with defining such measure, this step has not been performed yet. One promising direction in this regard is to use the natural measures of the embedding manifold. While in principle it can be seen as a breaking of the diffeomorphism invariance, the integral Hilbert spaces resulting from distinct embeddings (diffeomorphism gauge fixings) will be equivalent.

The presented technique may also be in principle applicable to the original (pioneering) formulation of LQG [32] where the Hamiltonian constraint operator is graph changing [33]. In that construction the Hamiltonian constraint operator always acts by adding new triangular (planar) loops and the new nodes are always three valent. This implies the existence of a certain "core" of the graph (edges connected to nodes of higher valence) which is preserved. These "cores" can then be used to define the equivalent of superselection sectors from graph preserving formulations. These "single sector" subspaces would be however still nonseparable and thus would require introduction of the integral structure on each sector separately. Building such "internal" integral structure would require in turn the detailed analysis on how precisely the construction of the Hamiltonian constraint 
specified above modifies the graph and is expected to be much more difficult than the construction in graph preserving formulations.

Apart from possible generalizations, it is important to point out one relevant feature of the construction introduced here. In principle, instead of following the loop quantization program strictly, one could regularize the Hamiltonian at the classical level (by introducing by hand a periodic potential) and quantize it in the standard Schrödinger representation. The final result would be identical to the one resulting from the point of view presented here. Whether such approach should be taken depends of the goals of the program. The alternative mentioned here gives rise to a consistent treatment deviating from LQG more than the standard polymeric quantization but still incorporating some of its central features. While without a direct reference to loop quantization the regularization of the Hamiltonian would not be justified, the present approach may be interesting at a phenomenological level. When exploring the consequences of the polymer quantization no such shortcut should be permitted and the precise construction of the separable Hilbert space must be provided. Skipping this step may lead to an incorrect description of the dynamical sector of the theory, as discussed in [17].

\section{ACKNOWLEDGMENTS}

This work has been supported by the Spanish MICINN and MINECO research Grants No. FIS2009-11893, No. FIS2011-30145-C03-02, No. FIS2012-34379 and the Consolider-Ingenio 2010 Program CPAN (CSD200700042), Chilean FONDECYT regular Grant No. 1140335 as well as by the National Center for Science $(\mathrm{NCN})$ of Poland research Grants No. 2012/05/E/ST2/03308 and No. 2011/02/A/ST2/00300. T. P. also acknowledges the financial support of UNAB via internal project No. DI-562-14/R.
[1] T. Thiemann, Modern Canonical Quantum General Relativity (Cambridge University Press, London, 2007).

[2] C. Rovelli, Quantum Gravity (Cambridge University Press, London, 2004).

[3] K. Giesel and T. Thiemann, Classical Quantum Gravity 27, 175009 (2010); K. Giesel and T. Thiemann, arXiv: 1206.3807

[4] M. Domagała, K. Giesel, W. Kamiński, and J. Lewandowski, Phys. Rev. D 82, 104038 (2010).

[5] V. Husain and T. Pawłowski, Phys. Rev. Lett. 108, 141301 (2012).

[6] J. F. Barbero G., J. Prieto, and E. J. Villaseñor, Classical Quantum Gravity 30, 165011 (2013).

[7] M. Bojowald, Living Rev. Relativity 11, 4 (2008); A. Ashtekar and P. Singh, Classical Quantum Gravity 28, 213001 (2011).

[8] A. Ashtekar and J. Lewandowski, Classical Quantum Gravity 21, R53 (2004).

[9] J. A. Zapata, J. Math. Phys. (N.Y.) 38, 5663 (1997).

[10] W. Fairbairn and C. Rovelli, J. Math. Phys. (N.Y.) 45, 2802 (2004).

[11] J. Lewandowski, A. Okołów, H. Sahlmann, and T. Thiemann, Commun. Math. Phys. 267, 703 (2006).

[12] L. Garay, M. Martín-Benito, and G. Mena Marugán, Phys. Rev. D 82, 044048 (2010).

[13] I. Agullo, A. Ashtekar, and W. Nelson, Phys. Rev. D 87, 043507 (2013).

[14] M. Fernández-Méndez, G. A. Mena Marugán, and J. Olmedo, Phys. Rev. D 86, 024003 (2012).

[15] S. S. Seahra, I. A. Brown, G. M. Hossain, and V. Husain, J. Cosmol. Astropart. Phys. 10 (2012) 041.

[16] W. Kamiński, J. Lewandowski, and T. Pawłowski, Classical Quantum Gravity 26, 245016 (2009).
[17] A. Kreienbuehl and T. Pawłowski, Phys. Rev. D 88, 043504 (2013).

[18] M. Reed and B. Simon, Methods of Modern Mathematical Physics Vol. II (Academic Press, New York, 1978).

[19] A. Corichi, T. Vukasinac, and J. A. Zapata, Phys. Rev. D 76, 044016 (2007); Classical Quantum Gravity 24, 1495 (2007).

[20] A. Ashtekar, M. Bojowald, and J. Lewandowski, Adv. Theor. Math. Phys. 7, 233 (2003).

[21] A. Ashtekar, T. Pawłowski, and P. Singh, Phys. Rev. D 74, 084003 (2006).

[22] A. Henderson and T. Pawłowski, "Bianchi I Universe Dynamics in LQC," (to be published).

[23] A. Ashtekar, T. Pawłowski, and P. Singh, Phys. Rev. D 73, 124038 (2006).

[24] A. Ashtekar, S. Fairhurst, and J. L. Willis, Classical Quantum Gravity 20, 1031 (2003).

[25] E. Bentivegna and T. Pawłowski, Phys. Rev. D 77, 124025 (2008).

[26] W. Kamiński and T. Pawłowski, Phys. Rev. D 81, 084027 (2010).

[27] G. A. Mena Marugán, J. Olmedo, and T. Pawłowski, Phys. Rev. D 84, 064012 (2011).

[28] A. Corichi and E. Montoya, Int. J. Mod. Phys. D 21, 1250076 (2012).

[29] A. Ashtekar, T. Pawłowski, P. Singh, and K. Vandersloot, Phys. Rev. D 75, 024035 (2007).

[30] M. Martín-Benito, D. Martín-de Blas, and G. A. Mena Marugán, Classical Quantum Gravity 31, 075022 (2014).

[31] K. Giesel and T. Thiemann, Classical Quantum Gravity 24, 2465 (2007).

[32] T. Thiemann, Classical Quantum Gravity 15, 839 (1998).

[33] T. Thiemann, Phys. Lett. B 380, 257 (1996). 